\title{
On the development of controlled stationary and travelling disturbances in the supersonic boundary layer of a swept wing
}

\author{
Gleb Kolosov ${ }^{1,2, *}$, Alexandra Panina ${ }^{1}$, and Alexander Kosinov ${ }^{1,2}$ \\ ${ }^{1}$ Khristianovich Institute of Theoretical and Applied Mechanics, SB RAS, Institutskaya Str. 4/1, \\ Novosibirsk 630090, Russia \\ ${ }^{2}$ Novosibirsk State University, Novosibirsk 630090, Russia
}

\begin{abstract}
Experimental data on the evolution of controlled stationary and travelling disturbances in a $3 \mathrm{D}$ supersonic boundary layer, over a $45^{\circ}$ swept-wing at Mach number 2.0, is presented. Travelling artificial disturbances were introduced in the boundary layer by periodical glow discharge, at a frequency of $20 \mathrm{kHz}$. Stationary disturbances were acquired by setting the roughness elements on the surface of the model. Spatialtemporal and spectral-wave characteristics of the wave train at the frequency $20 \mathrm{kHz}$, in the linear region of development, were obtained. It was found that the periodic modulation of mean flow can lead to the stabilization of unstable travelling disturbances in the supersonic boundary layer of a swept wing. These experiments have investigated the viability of using roughness elements to control laminar-turbulent transition.
\end{abstract}

\section{Introduction}

The transition to turbulence in swept-wing boundary layers has been the focus of considerable attention by researchers for more than 30 years $[1,2]$. Such research is of great practical relevance: an ability to control the transition process on airplane wings would lead to significant fuel savings in future supersonic transports. Unfortunately, a number of difficulties arise in studying the process of laminar-turbulent transition. An inherent property of swept-wing boundary layers is the presence of crossflow, which leads to the appearance of a new type of instability. Modern theory on transition mechanisms in threedimensional (3D) boundary layers gives four basic types of flow instabilities: (1) attachment line boundary layer instability on the swept leading edge; (2) crossflow instability; (3) centrifugal instability; and (4) possible instability of Tollmien-Schlichting waves. Their relative roles in laminar-turbulent transition strongly depends upon a variety of factors, such as the level of free stream disturbances, surface quality, Mach number, model geometry etc.

Most of the theoretical and experimental results on stability and transition of the 3D boundary layers have been obtained for subsonic flow. An important feature in such boundary layers is that flow becomes primarily unstable to both stationary vortices and

*Corresponding author: kolosov@itam.nsc.ru 
oblique travelling waves. Depending on the environmental conditions, two main scenarios for transition are possible [1]. One scenario concerns the domination of stationary disturbances (crossflow vortices), and can occur if the free-stream turbulence level is small [3]. In this case, stationary vortices, excited by surface roughness, are developed linearly and eventually saturate and become subject to high-frequency secondary instability. This results in laminar-flow breakdown $[4,5]$. An alternative scenario is that involving the domination of travelling disturbances, excited by the vortical content of the free stream turbulence. Observations of travelling instability modes are usually performed under controlled disturbance conditions, when wave packets are generated by a localized pulse [6]. It is important to note that a paper authored by Wasserman and Kloker [7], is the only paper to date to scrutinize crossflow breakdown by travelling modes.

A supersonic boundary layer is far more complex than an incompressible boundary layer and has been thoroughly investigated both experimentally and numerically. Several studies have been devoted to the evolution of travelling disturbances in 3D supersonic boundary layers, and it has been suggested that travelling disturbances are what dominates the transition $[8,9]$. In order to excite controlled travelling disturbances in a supersonic boundary layer, a glow discharge technique was developed at ITAM SB RAS [10]. Such a technique allows one to observe the linear development of a wave train with frequencies of $10 \mathrm{KHz}$ and $20 \mathrm{kHz}$, on a swept wing at Mach number $\mathrm{M}=2.0$. [11, 12]. Moreover, at ITAM SB RAS, the technique of pulsed excitation of a wave packet with a broad disturbance spectrum has been implemented to obtain wave characteristics for a wide range of frequencies [13].

Later stages of the transition are characterized by nonlinearity of the disturbance flow field, for example the nonlinear interaction between stationary and travelling waves. Using roughness elements on the model allows us to obtain stationary waves with specified characteristics. The effect of distributed roughness on transition control in supersonic boundary layers on swept-wing models was studied in $[14,15]$. It was shown that the periodic surface roughness near the leading edge may stabilize the flow or move the laminar-turbulent transition closer to the leading edge of the model. Note that the flow stabilization was caused by the influence of distributed roughness on the stationary crossflow instability mode.

The goal of this work is (1) to use periodical discharge to obtain wave characteristics of the most unstable disturbances in the swept-wing supersonic boundary layer at $\mathrm{M}=2.0$ and (2) to use distributed roughness elements to investigate the effects of transverse mean flow modulation on the generation and development of a wave packet with the same income flow parameters.

\section{Experimental setup and data processing}

The experiments were performed in a T-325 low-noise supersonic wind tunnel of ITAM SB RAS, at Mach number $M=2.0$ and unit Reynolds number $\mathrm{Re}_{1}=5 \times 10^{6} \mathrm{~m}^{-1}$.

Disturbances in the flow were measured with a constant-temperature hot-wire anemometer. Probes with tungsten wire, 10 microns in diameter and $1.5 \mathrm{~mm}$ long, were used. The value of overheat loading of the wire was set equal to 0.8 , and this resulted in $90 \%$ of the measured disturbances corresponding to mass-flow fluctuations. To move the probe along the $x, y$, and $z$ coordinates, traverse gear was used. The accuracy of determination of the probe position in the $x$ - and $z$-coordinates was $0.1 \mathrm{~mm}$, and $0.01 \mathrm{~mm}$ for $y$-coordinate.

A swept-wing model was used in the experiments. The model had a sweep angle of $45^{\circ}$ and a slightly blunted leading edge of radius $0.2 \mathrm{~mm}$. The swept wing had a $2.6 \%$ lensshape airfoil on the upper side and a flat surface on the bottom side, with a maximum 
thickness of $12 \mathrm{~mm}$. The curvature radius of the upper side of the model was approximately $4000 \mathrm{~mm}$. A source of localized artificial disturbances was built into the model, similar to that described by Kosinov et al. [10]. Controlled pulsations were generated by using highfrequency glow discharge in a chamber and were injected into the boundary layer through an aperture $0.4 \mathrm{~mm}$ in diameter, in the upper surface of the model. The source aperture was located at $x_{0}=56.6 \pm 0.3 \mathrm{~mm}$ from the leading edge on the line of symmetry of the model. The pulsation measurements were synchronized using glow discharge, which was ignited with a fundamental frequency of $20 \mathrm{kHz}$. AC and DC signals from CTA were written to the PC using a 12-bit ADC with sampling rate $750 \mathrm{kHz}$ and by DC voltmeter correspondingly. Four time traces, each of length 65536 points, were measured and written to file for each position of the hot wire.

Transverse modulation of the mean flow in the boundary layer was created using adhesive tape stickers. Roughness elements were located on the top surface of the model, parallel to the leading edge. The stickers were diamond in shape and their size was $3 \times 3$ $\mathrm{mm}$. Two variants of the experiment were conducted, corresponding to spacing between roughness elements of $6 \mathrm{~mm}$ and $12 \mathrm{~mm}$ respectively. The roughness elements each had a thickness of $60 \mathrm{~mm}$. The experimental set-up is presented in Figure 1. The spanwise measurements were made at a fixed normal distance from the model surface and at $y / \delta \approx$ const for each tranverse.

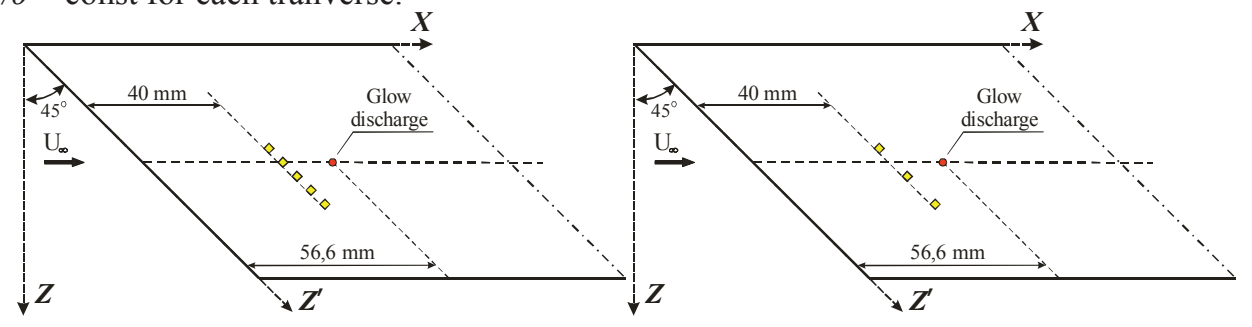

Fig. 1. Swept wing and set-up of the roughness elements; spacing of $6 \mathrm{~mm}$ between roughness elements (left), spacing of $12 \mathrm{~mm}$ between roughness elements (right)

The fluctuation time traces obtained, normalized by the mean voltage CTA output at each spatial measurement point, were transformed to mass flow fluctuations according to the relation $m\left(x^{\prime}, z^{\prime}, t\right)=e^{\prime}\left(x^{\prime}, z^{\prime}, t\right) /\left(Q \cdot E\left(x^{\prime}, z^{\prime}\right)\right)$. Here $e^{\prime}\left(x^{\prime}, z^{\prime}, t\right)$ is the fluctuation signal, $E\left(x^{\prime}, z^{\prime}\right)$ is the mean voltage from the CTA output, and $Q$ is the sensitivity of the probe to the mass flow fluctuations. Note that the probe measures the $x$-component of the mass flow fluctuation. The frequency-wave disturbance spectra at a fixed $x^{\prime}$-coordinate were calculated using the discrete Fourier transform of the following form:

$$
m_{f \beta^{\prime}}=\frac{\sqrt{2}}{T \delta_{n}} \sum_{j, k} m\left(z^{\prime}{ }_{j}, t_{k}\right) \exp \left[i\left(\beta^{\prime} z^{\prime}{ }_{j}-\omega t_{k}\right)\right] \Delta t \Delta z^{\prime}{ }_{j},
$$

where $T$ is the time-length of realization, $\beta^{\prime}$ is the spanwise component of the wave vector, and $\delta_{n}(=1 \mathrm{~mm})$ is the boundary layer thickness scale for normalizing the spectra in $\beta^{\prime}$. The Fourier transform in time was performed using the Hanning window. After performing the Fourier transform, the amplitude and phase of the controlled disturbances were calculating using:

$$
\begin{aligned}
& A_{f \beta^{\prime}}=\sqrt{\operatorname{Re}^{2}\left(m_{f \beta^{\prime}}\right)+\operatorname{Im}^{2}\left(m_{f \beta^{\prime}}\right)}, \\
& \Phi_{f \beta^{\prime}}=\arctan \left[-\operatorname{Im}\left(m_{f \beta^{\prime}}\right) / \operatorname{Re}\left(m_{f \beta^{\prime}}\right)\right] .
\end{aligned}
$$




\section{Results}

Results concerning the development of natural disturbances are presented in detail in [11, $12]$. Note that the source of the controlled disturbances is located in the linear region of the natural fluctuation development and, moreover, the frequency of the artificial pulsations $(f=20 \mathrm{kHz})$ falls within the frequency range for most unstable natural disturbances.

The controlled disturbance development on a smooth swept wing was measured in four different sections by means of displacing the anemometer probe along the $z^{\prime}$-coordinate, that is parallel to the leading edge of the model at $x=20,30,40$ and $50 \mathrm{~mm}$ from the discharge. The amplitude distributions of perturbations with frequency $f=20 \mathrm{kHz}$ in the spanwise direction are shown in Figure 2. One can see that in some places inside the wave packet, the amplitude grows downstream, whereas the main amplitude maximum is slowly displaced in the positive direction of the $z^{\prime}$-coordinate. Recall that the asymmetry of the amplitude distributions along the spanwise $z^{\prime}$-coordinate, relative to the position of the discharge $\left(z^{\prime}=0 \mathrm{~mm}\right)$, are associated with the presence of crossflow on the swept wing. The wave train is spread along the leading edge of the model in the direction opposite to the crossflow, and this is consistent with previously obtained results for the same Mach number (2.0) $[11,12]$.

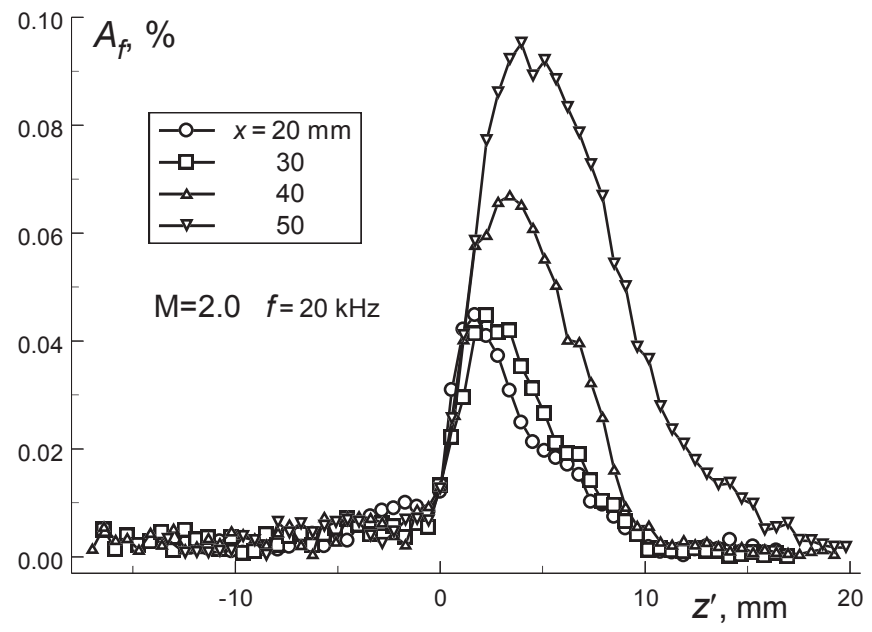

Fig. 2. Amplitude distributions of disturbances in the spanwise direction

Figure 3 shows the amplitude $\beta^{\prime}$-spectra of the controlled disturbances at frequency $20 \mathrm{kHz}$. Unlike in the flat plate case, the swept-wing $\beta^{\prime}$-spectra are asymmetric. The amplitude growth is observed in a wide range of panwise wavenumbers, but the amplitude maximum is located at $\beta^{\prime}=1.1 \mathrm{rad} / \mathrm{mm}$. This value corresponds to the linear peak in downstream wave train development. It should be noted that the traverses being considered don't belong to the nonlinear region of disturbance development. Thus one cannot expect the emergence of additional peaks in $\beta^{\prime}$-spectra other than the linear one. Growth over a wider range of $\beta^{\prime}$ is only observed for the cross section $x=50 \mathrm{~mm}$.

The results in the transverse-modulated supersonic boundary layer on a swept wing will now be discussed. Roughness element thickness was constant in all experiments and was 60 microns. Spacing between roughness elements was varied. All measurements were taken at the location of the maximum pulsations through the boundary layer, and at one of the edges of the tranverses where there was no mean flow modulation from the stickers. 


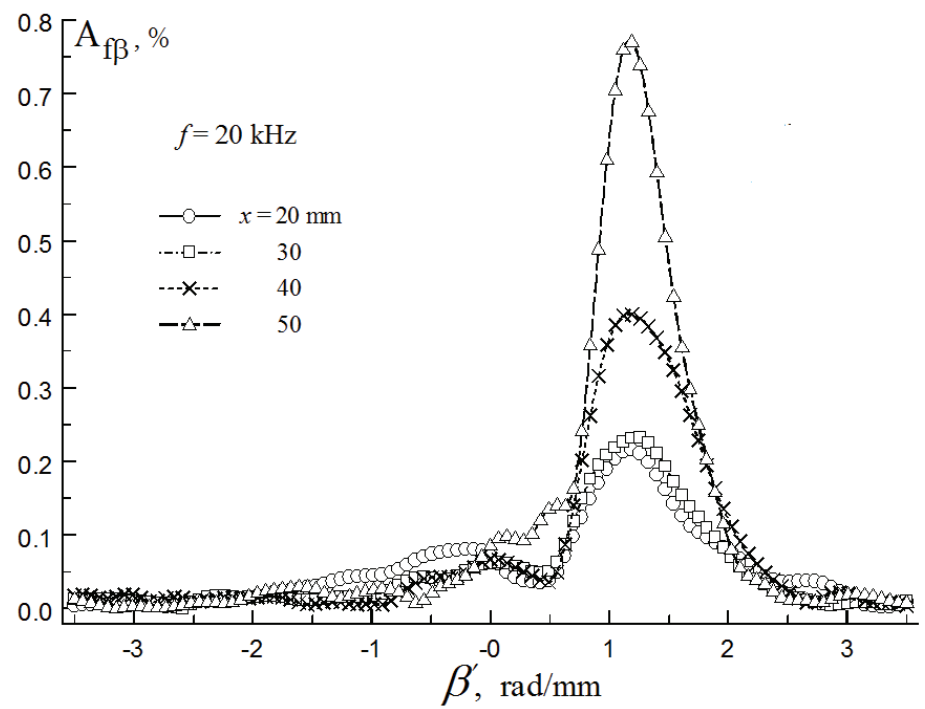

Fig. 3. Amplitude $\beta^{\prime}$-spectra of disturbances

Figure 4a shows the comparison of the mean flow modulation across the model in the initial section ( $x=14.14 \mathrm{~mm}$ from the discharge) for the cases of a smooth wing and swept wing with periodic stickers, with spacing between roughness elements of $6 \mathrm{~mm}$ and $12 \mathrm{~mm}$ respectively. It is clear from Figure 4a that the period of mean flow modulation corresponds to the period of roughness on the model surface. For the case of $6 \mathrm{~mm}$ spacing of roughness elements it was possible, in some region, to produce flow modulation resembling the sine function. Note that the disturbance source aperture on the model surface introduces an additional mean flow distortion, which can be observed near $z^{\prime} \approx 2 \mathrm{~mm}$ (see Figure 4a).

(a)

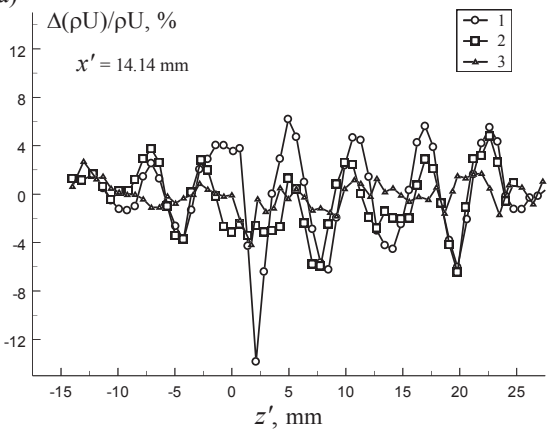

(b)

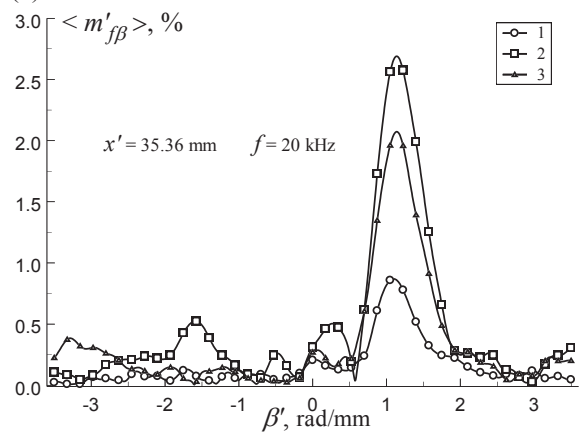

Fig. 4. (a) Mass flow variation; (b) amplitude $\beta^{\prime}$-spectra. 1 - spacing of $6 \mathrm{~mm}$ between roughness elements, 2 - spacing of $12 \mathrm{~mm}$ between roughness elements, 3 - smooth surface

The amplitude $\beta^{\prime}$-spectra of disturbances for the fundamental frequency $(f=20 \mathrm{kHz})$ at $x=35.4 \mathrm{~mm}$ is shown in Figure $4 \mathrm{~b}$. The results show that, in all cross sections, the perturbations showing most growth correspond to $\beta^{\prime} \approx 1 \mathrm{rad} / \mathrm{mm}$ wave number. This corresponds to the linear peak and it is in agreement with previously obtained results (see Figure 3). Note that the electrical power of the disturbance source was the same for all experiments. However, the data obtained shows that the relative receptivity of the boundary layer to nonstationary disturbances were significantly different. Also note that, in addition to the main linear peak, we can see a number of additional peaks. Their appearance cannot be explained by the linear theory, since they are the result of nonlinear wave interactions 
caused by the introduction of artificial stationary disturbances.

Figure 5 shows the relationship between transfer factor dependency and transverse wave number $\beta^{\prime}$, for the frequency $20 \mathrm{kHz}$. The results presented in Figure 5a show that at the initial stage (for the case of a swept wing with roughness elements separated by $12 \mathrm{~mm}$ ) the growth rate is greatest in the wide region, near $\beta^{\prime}=1 \mathrm{rad} / \mathrm{mm}$. However, at $x=35.36 \mathrm{~mm}$ from the disturbance source aperture, the growth rate of disturbances for the smooth wing is greater by a factor of approximately 1.5 times, compared to the case of the swept wing (for both values of roughness element separation) (see Figure 5b). Due to this experimental conclusion, it can be concluded that using microroughness enables the possibility of stabilizing disturbance development at the fundamental frequency in the boundary layer of a swept wing.
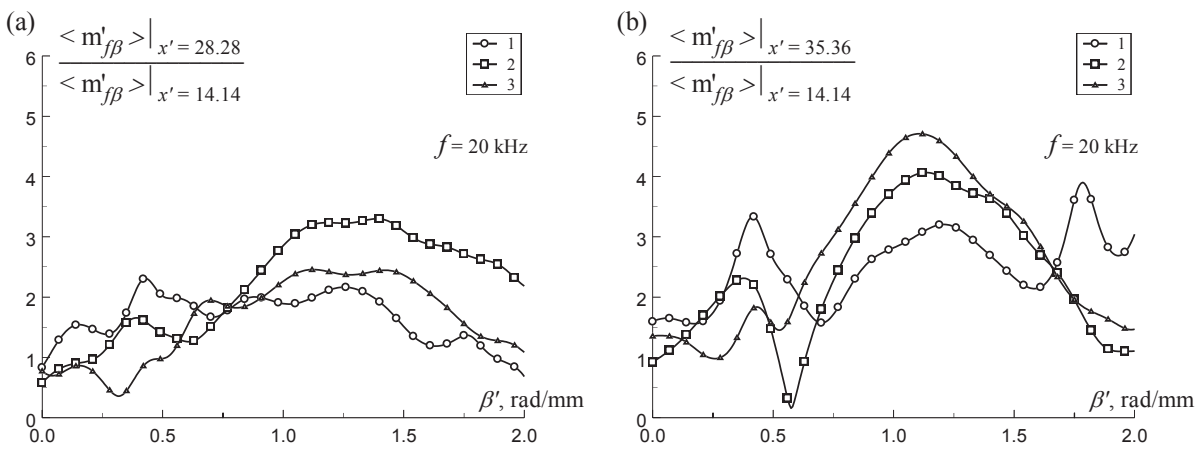

Fig. 5. Comparison of the transfer dependences over $\beta^{\prime}$ at the $20 \mathrm{kHz}$ frequency. 1 - spacing of $6 \mathrm{~mm}$ between roughness elements, 2 - spacing of $12 \mathrm{~mm}$ between roughness elements, 3 - smooth surface

\section{Conclusion}

An experimental study was conducted on the development of controlled travelling and stationary disturbances in a supersonic boundary layer of swept wing. This was performed at Mach 2.0 and unit Reynolds number $\mathrm{Re}_{1}=5 \times 10^{6} \mathrm{~m}^{-1}$. On the smooth swept wing, the region corresponding to linear stage of nonstationary disturbance evolution was examined. The amplitude distributions were shown to be spatially asymmetric, which can be attributed to the presence of a crossflow in 3D boundary layer. It was found that the amplitude maximum is located at $\beta^{\prime}=1.1 \mathrm{rad} / \mathrm{mm}$ for $20 \mathrm{kHz}$.

In experiments with roughness elements on swept wing it was shown that the variation of mean flow inside the boundary layer emerged that corresponds to the introduced stationary wave. It was determined that the roughness elements and their relative position can affect the downstream development of travelling pulsations. The experiments showed that the periodic modulation of mean flow by roughness elements can lead to the stabilization of travelling disturbances in the supersonic boundary layer of a swept wing. Therefore it is possible to utilize weak flow inhomogeneity, by using surface microroughness, to control supersonic boundary layer transition.

The work was supported by the Russian Foundation for Basic Research (Grant No. 16-31-00290).

\section{References}

1. H. Bippes, Prog. Aerospace Sci. 35, 363 (1999)

2. W. S. Saric, H. L. Reed, E. B. White, Annu. Rev. Fluid Mech. 35, 413 (2003) 
3. M.R. Malik, F. Li, C.-L. Chang, J. Fluid Mech. 268, 1 (1994)

4. D.I.A. Poll, J. Fluid Mech. 150, 329 (1985)

5. E.B. White, W.S. Saric, J. Fluid Mech. 525, 275 (2005)

6. V.R. Gaponenko, A.V. Ivanov, Y.S. Kachanov, Thermophys. Aeromechanics 2, 312 (1995)

7. P. Wasserman, M. Kloker, J. Fluid Mech. 483, 67 (2003)

8. L.N. Cattafesta III, V. Iyer, J.A. Masad, R.A. King, J.R. Dagenhart, AIAA J. 33, 2032 (1995)

9. F. Li, M. Choudhari, Theor. Comput. Fluid Dyn. 25, 65 (2011)

10. A.D. Kosinov, A.A. Maslov, S.G. Shevelkov, J. Fluid Mech. 219, 621 (1990)

11. Yu.G. Ermolaev, G.L. Kolosov, A.D. Kosinov, N.V. Semenov, Fluid Dynamics 49, 188 (2014)

12. A.D. Kosinov, G.L. Kolosov, N.V. Semionov, Yu.G. Yermolaev, Phys. Fluids 28, 064101 (2016)

13. A.A. Yatskikh, Yu.G. Ermolaev, A.D. Kosinov, N.V. Semionov, Thermophys. Aeromechanics 22, 17 (2015)

14. W.S. Saric, H.L. Reed, AIAA Paper 2002-0147, 10 (2002)

15. N.V. Semionov, A.D. Kosinov, Thermophys. Aeromechanics 14, 337 (2007) 